\title{
Comportamento ingestivo de bovinos em capim-piatã sob lotação intermitente em resposta a distintas alturas de entrada
}

\author{
Ingestive behavior cattle in piatã-grass under intermittent stocking with distinct entry \\ heights
}

\author{
MELO, Jnahtan Chaves ${ }^{1 *}$; ALEXANDRINO, Emerson ${ }^{2}$; PAULA NETO, Joaquim José \\ $\mathrm{de}^{3}$; REZENDE, José Messias de ${ }^{1}$; SILVA, André Augusto Marinho ${ }^{1}$; SILVA, Denise \\ Vieira $\mathrm{da}^{3}$; OLIVEIRA, Ana Kássia Ribeiro ${ }^{3}$
}

\footnotetext{
${ }^{1}$ Universidade Federal de Tocantins, Programa de Pós-Graduação em Ciência Animal Tropical, Araguaína, Tocantins, Brasil. Bolsista PNPD.

${ }^{2}$ Universidade Federal de Tocantins, Araguaína, Tocantins, Brasil.

${ }^{3}$ Universidade Federal de Tocantins, Curso de Zootecnia, Araguaína, Tocantins, Brasil.

*Endereço para correspondência: jonahtancmelo@hotmail.com.
}

\section{RESUMO}

O conhecimento da interface planta/animal no ecossistema pastagem é de fundamental importância quando se busca maximizar o desempenho produtivo do sistema. Nesse sentido, objetivou-se verificar se a altura de entrada em capim-piatã $(30,40$ e $50 \mathrm{~cm})$ interfere na qualidade da forragem, comportamento ingestivo, padrões de deslocamento e uso de estações alimentares de bovinos em duas condições de tempo, o pré-pastejo (zero dias de pastejo) e o pós-pastejo (sete dias de pastejo). As avaliações foram realizadas no período de 06h:30m as $18 \mathrm{~h}: 20 \mathrm{~m}$, no segundo e quarto ciclos de pastejo com animais que apresentavam 16 meses de idade e $239,8 \pm 25,7 \mathrm{~kg}$ de PV. As alturas de entrada não alteraram 0 comportamento ingestivo, mas houve efeito para a condição do pasto (pré e pós-pastejo). O número de intervalo de refeições foi maior aos $30 \mathrm{~cm}$ e menor no pré-pastejo. A maior taxa de bocados foi aos $30 \mathrm{~cm}$ e a menor aos $40 \mathrm{~cm}$, enquanto que, a redução da TB aos $50 \mathrm{~cm}$ em relação aos $30 \mathrm{~cm}$ reflete à dificuldade de manipular bocados. No pré-pastejo, maior número de estações por minuto foi verificada aos $40 \mathrm{~cm}$, enquanto no pós-pastejo foi aos $50 \mathrm{~cm}$. No pré-pastejo houve redução do tempo por refeição em relação ao pós-pastejo, refletindo a melhor estrutura do pasto nessa condição. A altura de entrada não alterou o comportamento ingestivo, entretanto, o tempo de pastejo modifica o comportamento ingestivo o padrão de deslocamento, busca e apreensão de forragem pelos animais, principalmente na condição póspastejo aos $50 \mathrm{~cm}$.

Palavras-chave: estrutura da planta, estações alimentares, ruminação, tempo ocioso, tempo de pastejo

\section{SUMMARY}

Knowledge of the interface plant and animal in the ecosystem grasslands is of fundamental importance when it seeks to maximize production performance of the system. That sense the objective was to verify if the input time piatã-grass (30, 40 and $50 \mathrm{~cm}$ ) interferes with the quality of the forage, feeding behavior, travel patterns and use of food cattle stations in two conditions of time, pregrazing (zero grazing days) and post-grazing (seven days of grazing). The evaluations were conducted in the period of $06 \mathrm{~h}: 30 \mathrm{~m} 18 \mathrm{~h}: 20 \mathrm{~m}$ in the second and fourth grazing cycles with animals with 16 months of age and $239.8 \pm 25.7 \mathrm{~kg}$ LW. The pregrazing heights did not change feeding behavior, but was no effect for the condition of the pasture (pre-grazing and post-grazing). The interval number of meals was higher at $30 \mathrm{~cm}$ and lower in pre-grazing. The highest bite rate was in $30 \mathrm{~cm}$ and $40 \mathrm{~cm}$ to the smallest, reflecting good condition to grazing, while reducing $\mathrm{TB}$ to $50 \mathrm{~cm}$ to $30 \mathrm{~cm}$ is related to the difficulty of using bits. In pre-grazing largest number of stations per minute was at $40 \mathrm{~cm}$, 
while in the post-grazing to $50 \mathrm{~cm}$. In pre-grazing decreased time per meal compared to the postgrazing, reflecting the best pasture structure in this condition. The entry height did not change feeding behavior, however, grazing time modify the feeding behavior standard of displacement, search and seizure of forage by the animals, especially in the post-grazing condition to $50 \mathrm{~cm}$.

Keywords: plant structure, feeding stations, rumination, idle time, grazing time

\section{INTRODUÇÃO}

A estrutura da planta forrageira, em muitas ocasiões, assume maior relevância que o seu valor nutritivo, e é importante para definir estratégias de manejo do pasto mais adequadas ao sistema de produção. A curto prazo o desempenho animal em pastagem está diretamente associado ao potencial instantâneo de ingestão dos bovinos, determinado pela facilidade de colheita da forragem, passíveis de fortes mudanças de acordo com a estrutura do dossel.

Longas jornadas destinadas à procura e seleção de forragem, com altas taxas de deslocamento e uso demasiado de número de estações alimentares, refletem a dificuldade de colheita de forragem pelo animal. Tal padrão de resposta resulta em gasto desnecessário de energia líquida de mantença que poderia ser destinada para ganho de peso.

De fato, em condições limitantes de pastejo os bovinos alteram $o$ comportamento ingestivo para manter o consumo de massa seca de forragem, mas nem sempre essas adaptações são suficientes para compensar a grande dificuldade de colheita que a estrutura impõe aos animais principalmente quando se aproxima a condição pós-pastejo, quando a oferta de lâmina foliar decresce.

Nesse sentido, estudos já foram desenvolvidos, apontando a altura do dossel forrageiro como importante característica, que modifica a taxa de ingestão instantânea e seletividade do alimento (MEZZALIRA et al., 2013), o número de movimentos mandibulares para colheita e processamento da forragem de acordo com a condição estrutural da planta e sua taxa de rebaixamento, ou seja, condição de pre-pastejo para pós-pastejo (FONSECA et al., 2013; MEZZALIRA et al., 2014).

O modelo de exploração com bases na condição fisiológica da planta para orientar o manejo da desfolhação e a etologia do pastejo animal, tende a verticalizar o sistema produtivo, principalmente quando se associa a utilização mais intensiva de insumos e novos cultivares como o capim-piatã, muito pouco estudado na região Norte do Brasil.

Dessa forma, o estabelecimento de metas de manejo para e planta forrageira, orientadas pela estrutura do dossel, com objetivo de potencializar o crescimento do pasto e a sua ingestão são de importância irrefutável no manejo da pastagem (CARVALHO et al., 2009). Nesse sentido, o presente estudo teve por objetivo verificar se a altura de entrada em capim-piatã $(30,40$ e $50 \mathrm{~cm})$ interfere na qualidade da forragem, comportamento ingestivo, padrões de deslocamento e uso de estações alimentares de bovinos em duas condições de tempo, o pré-pastejo (zero dias de pastejo) e o pós-pastejo (sete dias de pastejo).

\section{MATERIAL E MÉTODOS}

O experimento foi conduzido na Universidade Federal do TocantinsUFT, Campus Universitário de Araguaína-TO, com início em 20 de novembro de 2011 e término em 08 de maio de 2012, na Escola de Medicina Veterinária e Zootecnia, localizada a $07^{\circ} 12^{\prime} 28^{\prime \prime}$, Latitude Sul e 48 $12^{\prime} 26^{\prime \prime}$, 
Longitude Oeste, com altitude de $236 \mathrm{~m}$ em uma pastagem de capim-piatã (Urochloabrizantha cv. Piatã) estabelecida no ano de 2009.

$\mathrm{O}$ delineamento experimental foi o de blocos, com tratamentos em esquema de parcelas subdivididas no tempo. As alturas reais de entrada $(31,6 \pm 0,9$ $41,7 \pm 0,8$ e $52,1 \pm 1,1 \mathrm{~cm})$ constaram as parcelas principais e as condições de tempo, representadas pelo pré-pastejo (Tempo 1= zero dias de pastejo) e o póspastejo (Tempo $2=$ sete dias de pastejo) representaram as subparcelas, com duas repetições de piquetes e, 08 animais de prova para cada altura de entrada testada (04 animais por repetição de piquete) com um total de 24 animais teste na área experimental. Entretanto, as variáveis relacionadas à composição dos pastos de capim-piatã, tais como: massa de forragem no pré e pós-pastejo foi estimada pelo corte da forragem contida no interior de uma moldura metálica de $0,6 \mathrm{~m}^{2}(0,6 \times 1 \mathrm{~m})$ em dois sub-piquetes, dos vários que foram utilizados em função do período de descanso e de ocupação (Tabela 1), totalizando quatro repetições em cada altura estudada.

Tabela 1. Período de descanso (PD), período de ocupação e número de sub-piquetes utilizados para atingir as alturas testadas

\begin{tabular}{|c|c|c|c|c|}
\hline \multirow{2}{*}{ Alturas } & \multirow{2}{*}{ Ciclos } & PD & $\mathrm{PO}$ & \multirow{2}{*}{$\mathrm{N}^{\circ}$ sub-piqutes } \\
\hline & & \multicolumn{2}{|c|}{ (dias) } & \\
\hline \multirow{3}{*}{$30 \mathrm{~cm}$} & 2 & 25,8 & 6,5 & 4 \\
\hline & 4 & 21,5 & 6,5 & 4 \\
\hline & & $\widehat{\mathrm{X}}=23,7$ & 7,0 & $\widehat{\mathrm{X}}=4$ \\
\hline \multirow{3}{*}{$40 \mathrm{~cm}$} & 2 & 29,5 & 7,0 & 4 \\
\hline & 4 & 29,0 & 7,0 & 4 \\
\hline & & $\widehat{X}=29,2$ & 7,0 & $\widehat{X}=4,3$ \\
\hline \multirow{3}{*}{$50 \mathrm{~cm}$} & 2 & 37,8 & 7,4 & 5 \\
\hline & 4 & 39,2 & 6,5 & 6 \\
\hline & & $\widehat{\mathrm{X}}=39,9$ & 7,0 & $\widehat{\mathrm{X}}=5,7$ \\
\hline
\end{tabular}

$\mathrm{PD}=$ período de descanso; $\mathrm{PO}=$ período de ocupação em dias.

O clima da região, segundo a classificação de Köppen (1948) é AW - Tropical de verão úmido com estação chuvosa definida e período de estiagem no inverno. Durante o período experimental as condições de precipitação e temperatura média foram acessadas junto ao Instituto Nacional de Meteorologia (Figura 1).

Para estratificação da estrutura vertical do capim utilizou-se dois quadrados com volume $0,09 \mathrm{~m}^{3}(0,6 \times 1 \mathrm{~m} \times 0,15 \mathrm{~m})$, os quais permitiam sobreposição por meio de um suporte de fixação, no qual, retirava-se a moldura superior logo após o corte do primeiro estrato $(>30 \mathrm{~cm})$, para realização do corte dos demais estratos (30-15 e 15$0 \mathrm{~cm})$, dessa forma, foi possível quantificar a massa em três estratos $(0-15 ; 15-30$ e $>30 \mathrm{~cm}$ ). As amostras foram separadas manualmente nas frações lâminas foliares, colmo (colmo + bainha) e material morto. Para a determinação da massa seca, as amostras de cada componente morfológico foram secas em estufa de ventilação forçada a $65^{\circ} \mathrm{C}$ por 72 horas. Com os dados de massa seca de lâmina foliar e de colmo foi determinada a relação folha/colmo. 
A determinação do valor nutritivo foi feita em amostras de forragem retiradas por meio do método pastejo simulado, no pré e pós-pastejo. Foram determinados o teor proteína bruta $(\mathrm{PB})$
(SILVA \& QUEIROZ, 2002) e a digestibilidade in vitro da matéria seca (DIVMS), pela técnica de Tilley \& Terry (1963), adaptada para o uso do rúmen artificial, conforme Holden (1999).

Dias experimentais

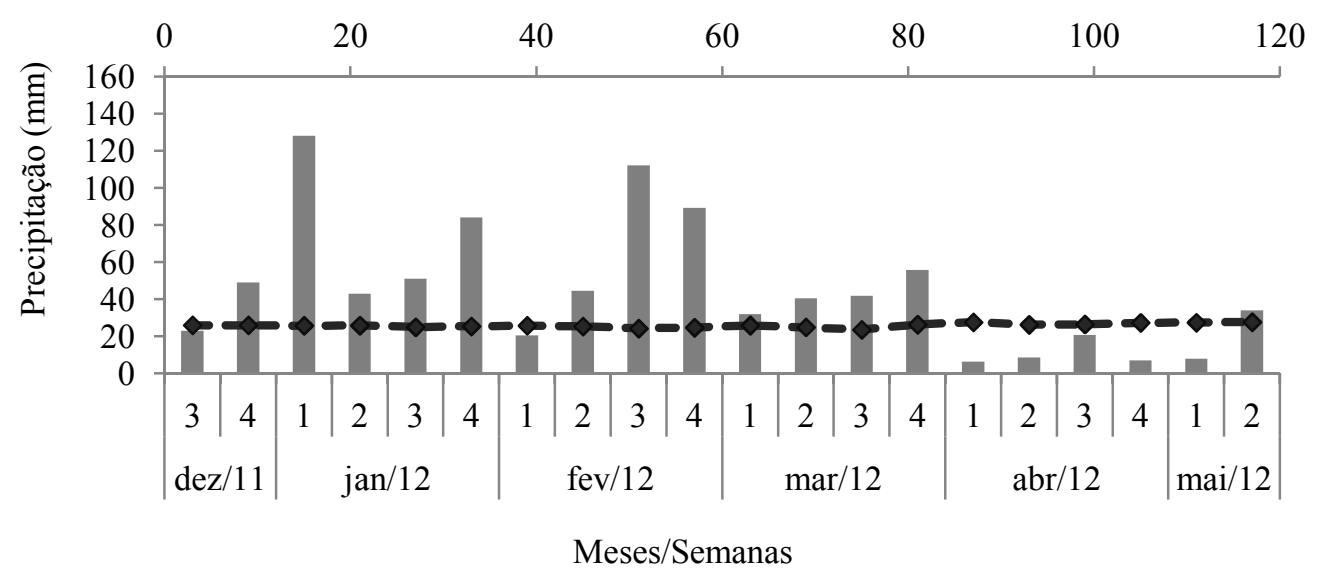

Figura 1. Precipitação (mm); $\cdots$ Temperatura média. Mensurados semanalmente na estação Agro-meteorológicado Instituto Nacional de Meteorologia (INMET, 2011/2012), localizada no Campus Universitário de Araguaína-TO

O comportamento ingestivo foi avaliado no segundo e quarto ciclos de pastejo por observações visuais em dois períodos contínuos de 12 horas, na condição pré e pós-pastejo, para os 24 animais de prova com peso vivo médio inicial de $239,8 \pm 25,7 \mathrm{~kg}$ de PV e 16 meses de idade. Os animais foram identificados com brincos numerados e para facilitar a visualização os animais foram pintados em diferentes partes do corpo (cernelha, lombo e anca).

No período de 12 horas as atividades de pastejo diurno, tempo de ruminação diurno, tempo em ócio foram devidamente marcadas em planilhas em intervalos de 10 minutos. O tempo de pastejo foi considerado como o tempo gasto pelos animais para seleção e apreensão do pasto, incluindo neste os períodos de tempo usados em deslocamentos para a seleção de forragem (GONÇALVES et al., 2009).
O tempo em ócio correspondeu ao período de descanso e a expressão de outras atividades. O tempo de pastejo (TP), de ruminação (TR) e ócio (TO) foram agrupados em intervalos de 180 minutos e os dados distribuídos percentualmente em diferentes períodos do dia $(1=6 \mathrm{~h} 30 \mathrm{~m}-09 \mathrm{~h}$ $20 \mathrm{~m} ; 2=9 \mathrm{~h} 30 \mathrm{~m}-12 \mathrm{~h} 20 \mathrm{~m} ; 3=12 \mathrm{~h} 30 \mathrm{~m}-$ 15 h 20m; 4= 15h 30m-18h 20m).

Durante os testes de pastejo quatro animais por piquete foram monitorados por dois avaliadores, que verificaram o número total de estações alimentares e o número total de passos dados pelo animal durante o teste de pastejo por meio de contador manual. A partir dos dados coletados foram calculadas as variáveis que compõem o processo de deslocamento e uso de estações alimentares conforme Gonçalves et al. (2009). O número de refeições e o número de intervalos de refeições foram obtidos por meio do 
controle das atividades dos animais, sendo que uma refeição foi considerada uma sequência de pastejo com no mínimo duas observações sucessivas (20 minutos) na atividade de pastejo e a interrupção do pastejo por qualquer outra atividade por mesmo período caracterizou o término da refeição e início do intervalo de refeições. As variáveis-respostas foram submetidas à Anova, para casos de medidas repetidas no tempo em que o tempo (Tempo $1=$ prépastejo "zero dias de pastejo" e Tempo $2=$ pós-pastejo "sete dias de pastejo") foi um fator testado como causa da variação. As médias foram calculadas utilizando LSMEANS e sua comparação realizada em nível de $5 \%$ de probabilidade pelo teste de Tukey. A escolha da matriz de covariância foi feita usando o Critério de informação de Akaike (AIC e BIC) (WOLFINGER, 1993). Ainda foi realizado teste de correlação simples entre as variáveis, sendo todas as comparações realizadas a $5 \%$ de probabilidade.

\section{RESULTADOS E DISCUSSÃO}

Ao longo do período experimental, em relação aos aspectos quantitativos, os pastos de capim-piatã não foram limitantes aos bovinos em pastejo para nenhuma das três estratégias de altura do pasto, pois os valores de massa seca total em todos os tratamentos (Figura 2a) ultrapassaram $2.000 \mathrm{~kg} \mathrm{ha}^{-1}$ de MS, valor sugerido como não limitante ao consumo de forragem e, consequentemente, ao desempenho animal, pois o ganho de peso médio de $0,801 \mathrm{~kg}$ animal ${ }^{-1}$ dia $^{-1}$ pode ser considerado elevado, o que reflete a eficácia do controle da altura de entrada como forma de moldar a estrutura do pasto e o seu valor nutritivo determinando facilidade de colheita de forragem de qualidade aos animais já que a proporção de lâmina foliar (PLF) esteve associada $(\mathrm{r}=0,82)$ ao teor de proteína bruta $(\mathrm{PB})$ na condição de pré-pastejo (Tabela 5).

Constatou-se proximidade entre as metas de manejo de altura, de 31,$6 ; 41,7$ e 52,1 $\mathrm{cm}$ para as metas pré-pastejo de 30, 40 e $50 \mathrm{~cm}$, respectivamente, em que o intervalo entre pastejo de 23,7; 29,2 e 39,9 dias para atingir as alturas de entrada nos pastos de capim-piatã determinaram variação na massa seca total (MST) e seus componentes morfológicos: massa seca de lâmina foliar (MSLF), massa seca de colmos (MSC), massa seca de material morto (MSMM) na condição pré-pastejo e massa seca de colmos (MSC) pós-pastejo (Figura 2b).

A modificação na estrutura do dossel, para atingir as metas de altura, alterou a proporção de componentes morfológicos nos estratos estudados (Figura 3). No estrato de $0-15 \mathrm{~cm}$, colmo e material morto contribuíram com a maior proporção entre os componentes morfológicos. Apesar disso, a proporção de lâmina (PLF) aos $30 \mathrm{~cm}$ foi maior em relação a 40 e $50 \mathrm{~cm}$. Os pastos de $30 \mathrm{~cm}$ apresentaram, no estrato $15-30 \mathrm{~cm}$, maior PLF e, aos 40 e $50 \mathrm{~cm}$, somente no estrato $>30$ foi possível essa visualização, o que caracterizou uma estrutura vertical diferenciada para as estratégias de altura pré-pastejo, que penalizou o ganho médio de peso diário aos $50 \mathrm{~cm}(30=893 ; 40=816$ e $50 \mathrm{~cm}=$ $\left.693 \mathrm{~g} \mathrm{animal}^{-1} \mathrm{dia}^{-1}\right)$, tal fator deve-se principalmente à redução da proporção de lâmina foliar (PLF) da condição de pré para pós-pastejo pois a PLF esteve associada $(\mathrm{r}=0,72)$ ao teor de PB póspastejo (Tabela 6).

Dessa forma, a medida que houve rebaixamento do pasto os componentes colmo e material morto com menor valor nutritivo apresentaram-se com maior proporção para essa condição de manejo, na qual ocorreu em média queda de quatro pontos percentuais na DIVMS (Tabela 1), a qual está associada $(\mathrm{r}=0,67)$ ao tempo por refeição (T/REF), pré-pastejo (Tabela 
5) e ao NB/E $(r=0,62)$ na condição de pós-pastejo (Tabela 6).

A intensificação na produção de colmos e material senescente aos $50 \mathrm{~cm}$ promoveu queda na qualidade da forragem, reduzindo a DIVMS e o teor de PB no pré e pós-pastejo (Tabela 1). Apesar da redução no teor de $\mathrm{PB}$ da altura de 30 para $40 \mathrm{~cm}$ em $16 \%$ (pré-pastejo), o GMD foi

(a)

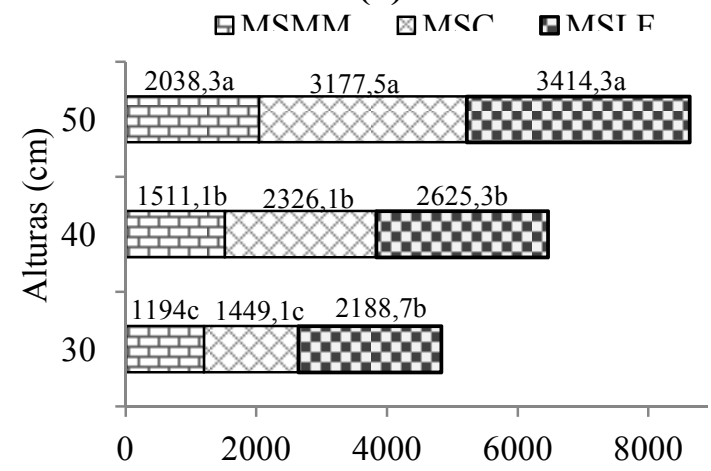

similar nessas estratégias, de 0,893 e $0,816 \mathrm{~kg}$ por dia, respectivamente. Entretanto, a redução da $\mathrm{PB}$, em $29 \%$, aos $50 \mathrm{~cm}$ esteve relacionada com redução de $22 \%$ no GMD, evidenciando que a modificação do GMD nas estratégias estudadas esteve mais relacionada com o valor nutritivo da forragem.

( $\mathrm{kg} / \mathrm{ha} \mathrm{MS)}$

Figura 2. (a)= massa seca de lâmina foliar (MSLF), colmos (MSC) e material morto (MSMM) pré-pastejo; (b) massa seca de lâmina foliar (MSLF), colmos (MSC) e material morto (MSMM) pós-pastejo em pastos de capim-piatã para diferentes alturas de entrada

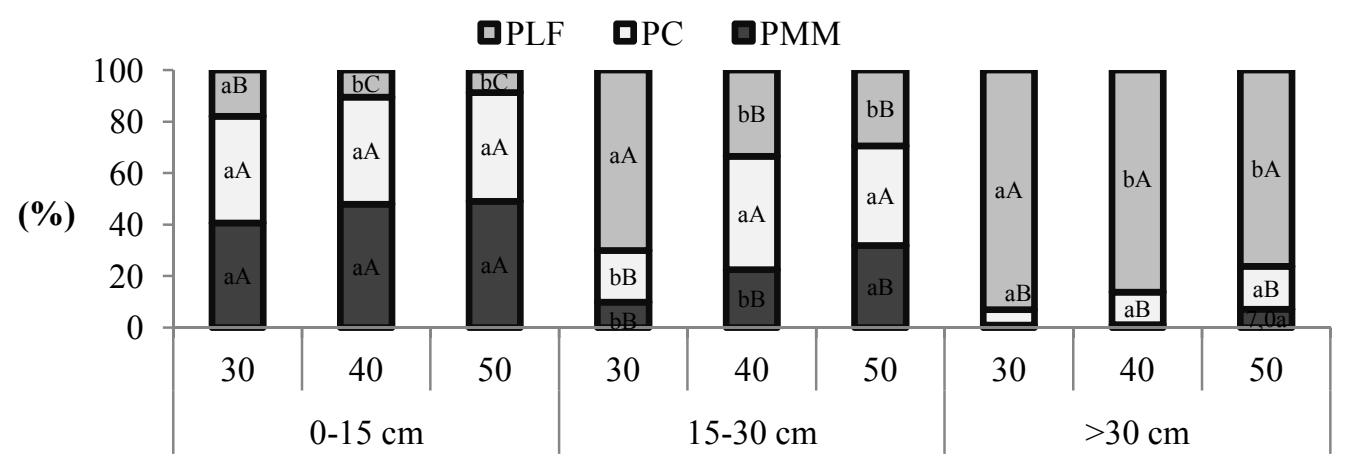

Estratificação vertical do Capim-Piatã (cm)

Figura 3. Proporção média dos componentes morfológicos para três alturas de entrada associados à estratificação da estrutura vertical do capim-piatã. Percentual de lâminas foliares (PLF); Percentual de colmo (PC); Percentual de material morto (PMM). Médias seguidas de letras distintas, minúsculas no estrato e maiúsculas entre estratos diferem entre si pelo teste de Tukey a 5\%, para o mesmo componente 
Tabela 1. Proteína bruta (PB) e digestibilidade in vitro da matéria seca (DIVMS) no pré e pós-pastejo em pastos de capim-piatã para diferentes alturas de entrada ${ }^{1}$

\begin{tabular}{lccc}
\hline \multirow{2}{*}{ Atributos do valor nutritivo (\% MS) } & \multicolumn{3}{c}{ Alturas $(\mathrm{cm})$} \\
\cline { 2 - 4 } & 30 & 40 & 50 \\
\hline PB* & $13,8^{\mathrm{aA}}$ & $11,6^{\mathrm{bA}}$ & $9,8^{\mathrm{cA}}$ \\
PB\# & $11,0^{\mathrm{aB}}$ & $10,9^{\mathrm{aA}}$ & $8,8^{\mathrm{bA}}$ \\
\hline DIVMS* & $66,8^{\mathrm{aA}}$ & $64,3^{\mathrm{abA}}$ & $62,3^{\mathrm{bA}}$ \\
DIVMS\# & $61,4^{\mathrm{abB}}$ & $62,2^{\mathrm{aA}}$ & $59,8^{\mathrm{bA}}$ \\
\hline
\end{tabular}

${ }^{\mathrm{T}}$ Médias seguidas por letras iguais, minúsculas na linha e maiúsculas na coluna, não diferem pelo Tukey a $5 \%$ de probabilidade de erro. *Condição de pré e \# pós-pastejo.

Entretanto, no pré e pós-pastejo a DIVMS decresceu apenas 6,7 e 2,6\%, respectivamente, de 30 para $50 \mathrm{~cm}$, dessa forma, apesar da grande variação no percentual de PB, observa-se que ela pouco afeta a DIVMS. Porém, decréscimos no valor nutritivo à medida que aumentou a altura do dossel de pastos de gramíneas tropicais já foram encontrados por (FLORES et al., 2008; EUCLIDES et al., 2009; CARLOTO et al. 2013), estando relacionados aos componentes fibrosos da planta e à lignificação dos componentes da parede celular que se torna mais espessa.

As alturas de entrada estudadas não alteram o comportamento ingestivo dos animais em pastejo, entretanto, o evoluir do tempo (condição de pré para póspastejo) modifica o comportamento ingestivo o padrão de deslocamento e uso de estações de alimentação, busca e apreensão de forragem pelos animais, principalmente aos $50 \mathrm{~cm}$.

Não houve efeito significativo da interação altura pré-pastejo $\mathrm{x}$ condição estrutural (Tabela 2) no tempo de pastejo, ruminação e ócio; somente a condição pré e pós-pastejo modificaram o TP e TO, com menores e maiores valores, respectivamente.
Esse fato está diretamente relacionado à estrutura do pasto na condição prépastejo que facilita a colheita, o que leva ao menor tempo por refeição já que proporcionalmente a planta não muda no pré-pastejo para os intervalos de altura.

Os animais efetuam maior número de refeições (NR) na condição pré-pastejo, com menor tempo por refeição (TR) e maior número de intervalos entre refeições (NIER), momento que os animais destinam seu tempo para outras atividades. Segundo Mezzalira et al. (2012), animais submetidos à baixa oferta de forragem (próximas a condição pós-pastejo no presente estudo) aumentam significativamente a sua duração de refeição, necessitando mais de 3 horas de pastejo e, os animais apresentaram intervalos mais curtos entre as refeições (30 minutos), e a relação entre a duração de alimentação e a duração do intervalo mostra que os animais destinam só um minuto para outras atividades a cada 5 minutos de pastejo. Assim, fica evidente que formas de manutenção de uma estrutura que conserve um nível ótimo de folhas nas plantas, principalmente no horizonte de pastejo, são formas hábeis para facilitar a colheita de forragem em pastos de capim-piatã. 
Tabela 2. Efeito da altura pré e pós-pastejo sobre o Tempo de pastejo diurno (TP), tempo de ruminação (TR) e tempo em ócio (TO), número de refeições (NR), número de intervalos entre refeições (NIER) e tempo por refeição (T/R) de bovinos em pastos de capim-piatã para diferentes alturas de entrada ${ }^{1}$

\begin{tabular}{|c|c|c|c|c|c|c|}
\hline \multirow{2}{*}{ Tempo 1 e Tempo 2} & \multicolumn{3}{|c|}{ Alturas $(\mathrm{cm})$} & \multirow{2}{*}{ Média } & \multirow{2}{*}{$\operatorname{Pr}>\mathrm{F}$} & \multirow{2}{*}{$\mathrm{CV} \%$} \\
\hline & 30 & 40 & 50 & & & \\
\hline \multicolumn{7}{|c|}{ Tempo de pastejo (minutos) } \\
\hline Pré-pastejo & 386,9 & 378,1 & 373,1 & $379,4^{\mathrm{B}}$ & \multirow{2}{*}{0,002} & \multirow[b]{2}{*}{15,0} \\
\hline Pós-pastejo & 431,9 & 442,5 & 470,0 & $448,1^{\mathrm{A}}$ & & \\
\hline \multicolumn{7}{|c|}{ Tempo de ruminação (minutos) } \\
\hline Média & $136,5^{\mathrm{a}}$ & $121,4^{\mathrm{a}}$ & $137,1^{\mathrm{a}}$ & 132,2 & 0,39 & 22,6 \\
\hline \multicolumn{7}{|c|}{ Tempo em ócio (minutos) } \\
\hline Pré-pastejo & 181,9 & 214,4 & 208,8 & $201,7^{\mathrm{A}}$ & \multirow{2}{*}{0,001} & \multirow{2}{*}{25,5} \\
\hline Pós-pastejo & 160,6 & 145,6 & 127,5 & $144,6^{\mathrm{B}}$ & & \\
\hline \multicolumn{7}{|c|}{ Número de refeições } \\
\hline Pré-pastejo & 6,8 & 6,6 & 7,0 & $6,8^{\mathrm{A}}$ & \multirow{2}{*}{0,05} & \multirow{2}{*}{22,8} \\
\hline Pós-pastejo & 6,6 & 5,9 & 5,9 & $6,2^{\mathrm{B}}$ & & \\
\hline \multicolumn{7}{|c|}{ Número de intervalos entre refeições } \\
\hline Pré-pastejo & 5,4 & 4,6 & 4,8 & $4,9^{\mathrm{A}}$ & 0,03 & 25,3 \\
\hline Pós-pastejo & 4,4 & 3,7 & 3,4 & $3,8^{\mathrm{B}}$ & - & - \\
\hline Média & $4,9^{\mathrm{a}}$ & $4,1^{\mathrm{b}}$ & $4,1^{\mathrm{b}}$ & 4,4 & 0,001 & - \\
\hline \multicolumn{7}{|c|}{ Tempo por refeição (minutos) } \\
\hline Pré-pastejo & 56,8 & 58,7 & 54,8 & $56,7^{\mathrm{B}}$ & 0,001 & 21,3 \\
\hline Pós-pastejo & 67,3 & 73,6 & 81,5 & $74,1^{\mathrm{A}}$ & - & - \\
\hline Média & $62,0^{\mathrm{a}}$ & $66,2^{\mathrm{a}}$ & $68,2^{\mathrm{a}}$ & 65,5 & 0,22 & - \\
\hline
\end{tabular}

${ }^{\mathrm{T}}$ Médias seguidas de letras minúsculas iguais na linha e maiúsculas na coluna não diferem estatisticamente pelo Tukey a $5 \%$ de probabilidade.

$\operatorname{Pr}>\mathrm{F}=$ probabilidade do erro tipo $1 ; \mathrm{CV} \%=$ coeficiente de variação.

O rebaixamento do pasto pela remoção contínua das lâminas foliares refletiu em menor densidade de lâmina pós-pastejo (Tabela 3), o que levou ao aumento do tempo destinado à atividade de pastejo pelos animais, em resposta a modificação na estrutura do dossel, ocasionada pela redução na proporção de folhas e elevação de colmos. Com o evoluir do período de ocupação os animais deixam de encontrarem lâmina foliar, preferencialmente selecionada, ocorrendo tal padrão de resposta quando o rebaixamento chega próximo a $40 \%$ da altura inicial do pasto (FONSECA et al., 2013). Após esse momento, uma segunda camada é exposta aos animais dificultando a colheita de forragem, principalmente aos $50 \mathrm{~cm}$, onde os animais gastaram mais de $8 \%$ de tempo na atividade se comparado aos $30 \mathrm{~cm}$ para satisfazer uma condição mínima de ingestão de forragem.

Observou-se que não houve modificação na proporção de lâmina foliar e, sim, de colmos, nas maiores alturas de entrada, o que promoveu com a evolução dos ciclos de pastejo, aumento da MST póspastejo em decorrência da rejeição desse componente pelos animais. Segundo Casagrande et al. (2010), quando ocorre aumento na proporção de colmos na parte superior do dossel, dificilmente a altura do pasto reduz e, nessa condição, o componente 'colmo' se torna um impedimento físico ao processo de pastejo, pela dificuldade em que os animais encontram para consumi-los. Dessa forma, os animais destinam maior tempo na atividade de pastejo na condição pós-pastejo. 
Tabela 3. Densidade de lâmina foliar (DSLF) e densidade de colmo (DSCO) em pastos de capim-piatã na condição pré e pós-pastejo para diferentes alturas ${ }^{1}$

\begin{tabular}{lcccccc}
\hline \multirow{2}{*}{ Tempo 1 e Tempo 2 } & 30 & 40 & Alturas $(\mathrm{cm})$ & Média & \multirow{2}{*}{ Pr> F } & \multirow{2}{*}{ CV \% } \\
\cline { 2 - 4 } & \multicolumn{7}{c}{ DSLF $\left(\mathrm{kg} \mathrm{cm}^{-1} \mathrm{ha}^{-1}\right)$} \\
\hline Pré-pastejo & 69,1 & 64,1 & 64,2 & $65,8^{\mathrm{A}}$ & \multirow{2}{*}{0,001} & \multirow{2}{*}{18,7} \\
Pós-pastejo & 27,4 & 29,5 & 26,1 & $27,7^{\mathrm{B}}$ & & \\
\hline \multicolumn{7}{c}{ DSCOL $\left(\mathrm{kg} \mathrm{cm}^{-1} \mathrm{ha}^{-1}\right)$} \\
\hline Pré-pastejo & 45,9 & 56,1 & 61,0 & $54,3^{\mathrm{B}}$ & \multirow{2}{*}{0,001} & \multirow{2}{*}{15,64} \\
Pós-pastejo & 48,2 & 62,5 & 74,6 & $61,7^{\mathrm{A}}$ & 0.001 & \\
Média & $47,0^{\mathrm{b}}$ & $59,3^{\mathrm{ab}}$ & $67,8 \mathrm{a}$ & 57,9 & 0,001 & \\
\hline
\end{tabular}

${ }^{1}$ Médias seguidas de letras minúsculas iguais na linha e maiúsculas iguais na coluna não diferem estatisticamente pelo Tukey a $5 \%$ de probabilidade do erro; $C V \%=$ coeficiente de variação.

Ao observar a distribuição das atividades expressas em termos percentuais da atividade em intervalos contínuos de $3 \mathrm{~h}$ 00m (Figura 4) na condição pré-pastejo, observou-se que somente no início da manhã e final da tarde, grande parte do tempo dos animais é destinada a atividade de pastejo, independente da altura pré-pastejo. Tais atitudes comportamentais podem estar relacionadas ao total de forragem ofertada nas primeiras horas de pastejo, com maiores proporções de folhas, além de boas condições ambientais que tendem a estimular o pastejo.

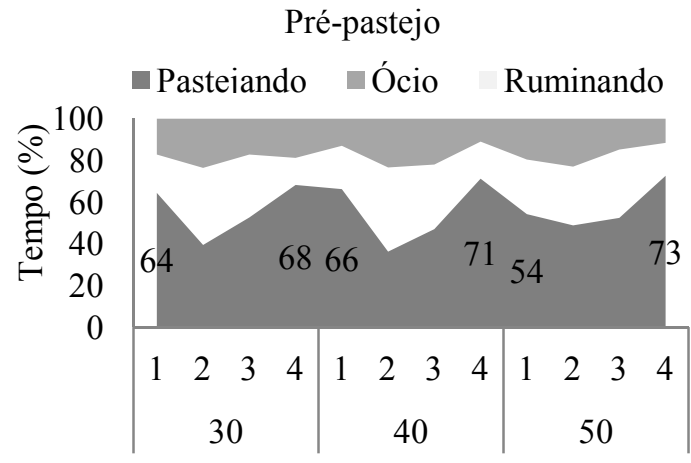

Alturas de entrada (cm)
Pós-pastejo

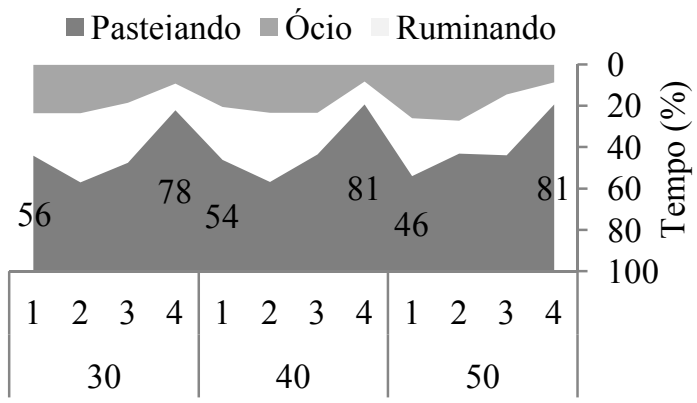

Alturas de entrada $(\mathrm{cm})$

Figura 4. Distribuição percentual das atividades diurnas por novilhos de recria, agrupadas em quatro tempos $(1=06 \mathrm{~h} 30 \mathrm{~m}-09 \mathrm{~h} 20 \mathrm{~m} ; 2=09 \mathrm{~h} 30 \mathrm{~m}-12 \mathrm{~h}$ $20 \mathrm{~m} ; 3=12 \mathrm{~h} 30 \mathrm{~m}-15 \mathrm{~h} \mathrm{20m} ; 4=15 \mathrm{~h} \mathrm{30m-18h} \mathrm{20m)}$

No pós-pastejo observou-se mesmo padrão de resposta às alturas de entrada (Figura 4), todavia, menor percentual de tempo foi gasto nas primeiras horas de pastejo em relação ao último turno de pastejo, com valores de $56 v s$ 78, $54 v s$ 81 e 46 vs $81 \%$, para as alturas de 30, 40 e $50 \mathrm{~cm}$, respectivamente, sendo esse padrão comportamental definido pela memória experiência-aprendizagem do animal como relatados por Griffiths et al. (2003), onde os animais ao perceberem proximidade da condição de rotação dos pastos, na expectativa de colher forragem em quantidade $\mathrm{e}$ qualidade diminuem a atividade de 
pastejo. Entretanto, com a evolução dos turnos de pastejo os animais aumentaram o tempo de pastejo e, novamente, no final da tarde, quase todo tempo foi destinado à atividade de pastejo, com 78 , 81 e $81 \%$ para as alturas de 30,40 e 50 $\mathrm{cm}$, respectivamente, uma forma de compensar total ou parcialmente $\mathrm{o}$ tempo destinado a outras atividades de forma a maximizar a ingestão de massa seca que é limitante no pós-pastejo, principalmente na maior altura de manejo (Figura 4).
A taxa de bocados (TB) foi influenciada somente pela altura do dossel, com maior valor para a altura de $30 \mathrm{~cm}$, seguidos da altura de 50 e $40 \mathrm{~cm}$ (Tabela4). Os novilhos efetuaram maior número de bocados por estação alimentarnos nos pastos de $30 \mathrm{~cm}$, seguidos da altura de $50 \mathrm{~cm}$ e por último a de $40 \mathrm{~cm}$. O número de bocados por estação alimentar (NBE) também foi responsivo as condições estruturais do dossel com maior número de bocados por estação no pré-pastejo (Tabela4).

Tabela 4. Padrões de deslocamento e uso de estações alimentares por bovinos em pastos de Capim-Piatã com diferentes alturas de entrada ${ }^{1}$

\begin{tabular}{|c|c|c|c|c|c|c|}
\hline \multirow{2}{*}{ Tempo 1 e Tempo 2} & \multicolumn{3}{|c|}{ Alturas $(\mathrm{cm})$} & \multirow{2}{*}{ Média } & \multirow{2}{*}{$\operatorname{Pr}>F$} & \multirow{2}{*}{$\mathrm{CV} \%$} \\
\hline & 30 & 40 & 50 & & & \\
\hline \multicolumn{7}{|c|}{ Taxa de Bocados ( $\mathrm{n}^{\circ}$ bocados por minuto) } \\
\hline Média & $38,9^{\mathrm{a}}$ & $30,5^{\mathrm{c}}$ & $35,2^{\mathrm{b}}$ & 35,0 & 0,001 & 16,8 \\
\hline \multicolumn{7}{|c|}{ Número de Bocados por Estação Alimentar } \\
\hline Pré-pastejo & 9,5 & 5,2 & 8,2 & $7,6^{\mathrm{A}}$ & 0,001 & \\
\hline Pós-pastejo & 6,9 & 6,8 & 6,0 & $6,6^{\mathrm{B}}$ & & 18,9 \\
\hline Média & $8,2^{\mathrm{a}}$ & $6,1^{\mathrm{b}}$ & $7,1^{\mathrm{ab}}$ & 7,1 & 0,001 & \\
\hline \multicolumn{7}{|c|}{ Número de Estações por Minuto } \\
\hline Pré-pastejo & $4,1^{\mathrm{bB}}$ & $5,6^{\mathrm{aA}}$ & $4,2^{\mathrm{bB}}$ & 4,6 & \multirow{2}{*}{0,001} & \multirow{2}{*}{14,9} \\
\hline Pós-pastejo & $5,7^{\mathrm{bA}}$ & $5,0^{\mathrm{bA}}$ & $6,1^{\mathrm{aA}}$ & 5,6 & & \\
\hline \multicolumn{7}{|c|}{ Taxa de Deslocamento (passos por minuto) } \\
\hline Pré-pastejo & 6,1 & 7,0 & 7,3 & $6,8^{\mathrm{B}}$ & \multirow{2}{*}{0,04} & \multirow{3}{*}{17,7} \\
\hline Pós-pastejo & 7,1 & 6,8 & 8,1 & $7,5^{\mathrm{A}}$ & & \\
\hline Média & $6,6^{\mathrm{b}}$ & $6,9^{\mathrm{b}}$ & $7,7^{\mathrm{a}}$ & 7,1 & 0,001 & \\
\hline \multicolumn{7}{|c|}{ Tempo por Estação Alimentar (segundos) } \\
\hline Pré-pastejo & 15,9 & 11,7 & 15,6 & $14,4^{\mathrm{A}}$ & \multirow{2}{*}{0,001} & \multirow{3}{*}{14,4} \\
\hline Pós-pastejo & 11,2 & 12,8 & 10,9 & $11,6^{\mathrm{B}}$ & & \\
\hline Média & $13,6^{\mathrm{a}}$ & $12,2^{b}$ & $12,7^{\mathrm{ab}}$ & 12,8 & 0,01 & \\
\hline
\end{tabular}

${ }^{1}$ Médias seguidas de letras minúsculas iguais na linha e maiúsculas iguais na coluna não diferem estatisticamente pelo Tukey a 5\% de probabilidade. $\operatorname{Pr}>\mathrm{F}=$ probabilidade do erro tipo 1; $\mathrm{CV} \%=$ coeficiente de variação.

A proporção média de rebaixamento da altura da condição pré-pastejo para póspastejo representou cerca de 26; 39 e $45 \%$ para os pastos de 30,40 e $50 \mathrm{~cm}$, sugerindo que a maior altura pré-pastejo dificulta a colheita de forragem, visto que Fonseca et al. (2013) demonstram que a massa do bocado e taxa de bocados mantiveram-se constantes e não limitante à seleção de 32 a 39\% de rebaixamento da altura, respectivamente, diminuindo linearmente seus valores com o aumento 
da proporção do rebaixamento até $80 \%$ para o Sorghum bicolor patejado por bovinos. A redução na taxa de bocados em pastos com estruturas comprometidas como na altura de $50 \mathrm{~cm}$ são devido à dificuldade do animal em manipular os bocados colhidos, resultando em aumento no tempo por bocado (PALHANO et al., 2007; DIFANTE et al., 2009; FONSECA et al., 2013).

$\mathrm{O}$ número de estações alimentares por minuto (Tabela4) foi influenciado pelas alturas e as condições, com maior número de estações no pré-pastejo para a altura de $40 \mathrm{~cm}\left(5,6\right.$ estações minuto $\left.{ }^{-1}\right) \mathrm{e}$ pós-pastejo para $50 \mathrm{~cm}(6,1$ estações minuto $\left.^{-1}\right)$, valores próximos aos encontrados por Oliveira Neto et al. (2013) em pastagens com duas fases de crescimento; vegetativa $(7,2$ estações minuto $\left.^{-1}\right)$ ou florecimento $(6,2$ estações minuto $\left.^{-1}\right)$. Segundo Carvalho et al. (2008) o tempo de permanência na estação alimentar está diretamente relacionado à quantidade de forragem disponível e à presença de colmos e material morto no horizonte de pastejo, são fatores limitantes da profundidade dos bocados. Nessas condições, a relação custo-benefício em continuar explorando a mesma estação alimentar torna-se inviável em nível de formação de bocados.

Somente para altura de $40 \mathrm{~cm}$ não foi detectado efeito da condição estrutural, sendo a resposta da estratégia de 30 e $50 \mathrm{~cm}$ similar, ocorrendo aumento do número de estações do pré para o póspastejo, podendo ser possível que os animais tenham escolhido novos patchs mais próximos, devido a percepção de melhores oportunidades de consumo em outros locais que podem ter beneficiado mudanças de estações alimentares nos pastos de $40 \mathrm{~cm}$, resultando números iguais de estações alimentares entre as condições pré e pós-pastejo, corroborando com Teixeira et al. (2011) ao encontrarem mesmo padrão de respostas em pastos de Brachiaria decumbens com alta disponibilidade de forragem.

A taxa de deslocamento e o tempo por estação alimentar foram responsivos as alturas e as condições estruturais (Tabela 4), com maior taxa de deslocamento para o pós-pastejo e maior tempo por estação no pré-pastejo. Segundo Gregorini et al. (2011), com a redução da massa de forragem em pastagens de trigo, os animais procuram efetuar bocados mais rentáveis, tentando manter uma taxa constante de consumo, processo que deprime a taxa de ingestão com o evoluir do período de pastejo, aumentando o número de estações visitadas a distância percorrida pelo animal e o número de bocados por estação alimentar.

Estes resultados estão de acordo com Facuri et al. (2014) ao demosntrarem que a suplementação do pasto com glicerina reduz o tempo de pastejo e aumenta o tempo em outras atividades, pois o glicerol atua como precursor gliconeogênico, mostrando a importancia do status energético a nível metabolico, que nesse caso, aumentou a saciedade dos animais e, além disso, esteve relacionado com a melhoria do uso da FDNe DIVMS melhorando a eficiencia de alimentação e ruminação. Dessa forma, é possível afirmar que os animais não atendem suas necessidades energéticas aos $50 \mathrm{~cm}$ e por isso aumentam seu deslocamente e o número de estações alimentares na condição de pós-pastejo o que pode ter suprido em parte o consumo de forragem e a demanda por energia pois os animais não aumentaram o tempo de pastejo no intervalo de altura estudado, entretanto, esse mecanismo adaptativo de escolha e uso de estacões alimentares não foi suficiente para manter constante o ganho medio diário na estratégia de manejo do pastejo aos 50cm (Tabelas 5 e 6). 
Tabela 5. Matriz de correlação simples entre variáveis para a composição química da forragem e comportamento ingestivo de animais em pastejo de capim-piatã na condição pré-pastejo (Tempo 1= zero dias de pastejo)

\begin{tabular}{|c|c|c|c|c|c|c|c|c|c|c|c|c|c|c|}
\hline & & DIVIMS & PB & TP & TO & TR & TB & $\mathrm{NB} / \mathrm{E}$ & NEST/M & TD & $\mathrm{T} / \mathrm{E}$ & NR & NIER & T/REF \\
\hline \multirow[t]{2}{*}{$\mathrm{PB}^{1}$} & $\mathrm{R}$ & 0,73 & - & - & - & - & - & - & - & - & - & - & - & - \\
\hline & $\mathrm{P}$ & $* *$ & - & - & - & - & - & - & - & - & - & - & - & - \\
\hline \multirow[t]{2}{*}{$\mathrm{TP}$} & $\mathrm{R}$ & 0,27 & 0,31 & - & - & - & - & - & - & - & - & - & - & - \\
\hline & $\mathrm{P}$ & ns & ns & - & - & - & - & - & - & - & - & - & - & - \\
\hline \multirow[t]{2}{*}{ TO } & $\mathrm{R}$ & $-0,59$ & $-0,50$ & $-0,55$ & - & - & - & - & - & - & - & - & - & - \\
\hline & $\mathrm{P}$ & $*$ & ns & ns & - & - & - & - & - & - & - & - & - & - \\
\hline \multirow[t]{2}{*}{$\mathrm{TR}$} & $\mathrm{R}$ & 0,28 & 0,16 & $-0,25$ & 0,28 & - & - & - & - & - & - & - & - & - \\
\hline & $\mathrm{P}$ & ns & ns & ns & ns & - & - & - & - & - & - & - & - & - \\
\hline \multirow[t]{2}{*}{ TB } & $\mathrm{R}$ & 0,25 & 0,46 & 0,12 & 0,25 & 0,17 & - & - & - & - & - & - & - & - \\
\hline & $\mathrm{P}$ & ns & $\mathrm{ns}$ & $\mathrm{ns}$ & $\mathrm{ns}$ & ns & - & - & - & - & - & - & - & - \\
\hline \multirow{2}{*}{$\mathrm{NB} / \mathrm{E}$} & $\mathrm{R}$ & 0,24 & 0,35 & 0,07 & 0,24 & 0,34 & 0,94 & - & - & - & - & - & - & - \\
\hline & $\mathrm{P}$ & ns & $\mathrm{ns}$ & $\mathrm{ns}$ & $\mathrm{ns}$ & ns & $* *$ & - & - & - & - & - & - & - \\
\hline \multirow[t]{2}{*}{ NEST/M } & $\mathrm{R}$ & $-0,23$ & $-0,13$ & $-0,09$ & $-0,47$ & $-0,47$ & $-0,66$ & $-0,85$ & - & - & - & - & - & - \\
\hline & $\mathrm{P}$ & ns & ns & ns & ns & ns & $* *$ & $* *$ & - & - & - & - & - & - \\
\hline \multirow[t]{2}{*}{ TD } & $\mathrm{R}$ & $-0,46$ & $-0,61$ & $-0,47$ & $-0,06$ & $-0,06$ & $-0,43$ & $-0,44$ & $-0,46$ & - & - & - & - & - \\
\hline & $\mathrm{P}$ & na & $*$ & ns & ns & ns & ns & ns & ns & - & - & - & - & - \\
\hline \multirow[t]{2}{*}{$\mathrm{T} / \mathrm{E}$} & $\mathrm{R}$ & 0,22 & 0,29 & 0,20 & 0,43 & 0,43 & 0,67 & 0,82 & 0,22 & $-0,64$ & - & - & - & - \\
\hline & $\mathrm{P}$ & ns & ns & ns & ns & ns & $* *$ & $* *$ & ns & $* *$ & - & - & - & - \\
\hline \multirow[t]{2}{*}{ NR } & $\mathrm{R}$ & $-0,41$ & 0,008 & 0,47 & $-0,14$ & $-0,14$ & 0,19 & 0,07 & $-0,40$ & $-0,07$ & 0,09 & - & - & - \\
\hline & $\mathrm{P}$ & ns & ns & ns & ns & ns & ns & ns & ns & ns & ns & - & - & - \\
\hline \multirow[t]{2}{*}{ NIER } & $\mathrm{R}$ & 0,26 & 0,49 & 0,10 & 0,45 & 0,45 & 0,70 & 0,69 & 0,26 & $-0,38$ & 0,54 & 0,10 & - & \\
\hline & $\mathrm{P}$ & ns & ns & ns & ns & ns & $* *$ & $* *$ & ns & ns & ns & ns & - & - \\
\hline \multirow[t]{2}{*}{$\mathrm{T} / \mathrm{REF}$} & $\mathrm{R}$ & 0,67 & 0,26 & $-0,06$ & $-0,13$ & $-0,13$ & $-0,23$ & $-0,22$ & 0,67 & $-0,13$ & $-0,18$ & -072 & $-0,36$ & - \\
\hline & $\mathrm{P}$ & $* *$ & ns & ns & ns & ns & ns & ns & $* *$ & ns & ns & $* *$ & ns & - \\
\hline \multirow[t]{2}{*}{ PLF } & $\mathrm{R}$ & 0,49 & 0,82 & 0,54 & 0,18 & 0,18 & 0,57 & 0,54 & 0,49 & $-0,70$ & 0,56 & 0,24 & 0,69 & $-0,07$ \\
\hline & $\mathrm{P}$ & $\mathrm{ns}$ & $* *$ & ns & ns & ns & $*$ & $*$ & ns & $* *$ & $*$ & ns & $* *$ & ns \\
\hline
\end{tabular}

${ }^{1}$ Digestibilidade in vitro da matéria seca (DIVIMS), proteína bruta (PB), proporção de lâmina foliar (PLF), tempo de pastejo (TP), tempo de ruminação (TR) e tempo em ócio (TO), taxa de bocados (TB), número de bocados por estação (NB/E), número de estações por minuto (NEST/M), taxa de deslocamento (TD), tempo por estação (T/E) número de refeições $(\mathrm{NR})$, número de intervalos entre refeições (NIER) e tempo por refeição $(\mathrm{T} / \mathrm{REF})$ de bovinos em pastos de capim-piatã. $\mathrm{R}=$ coeficiente de correlação, $\mathrm{P}=* * \mathrm{e} *$ significativo a 1 e $5 \%$ de probabilidade, respectivamente. 
Tabela 6. Matriz de correlação simples entre variáveis para a composição química da forragem e comportamento ingestivo de animais em pastejo de capim-piatã na condição pós-pastejo (Tempo 2= sete dias de pastejo)

\begin{tabular}{|c|c|c|c|c|c|c|c|c|c|c|c|c|c|c|}
\hline & & DIVIMS & $\mathrm{PB}$ & $\mathrm{TP}$ & TO & TR & TB & $\mathrm{NB} / \mathrm{E}$ & NEST/M & TD & $\mathrm{T} / \mathrm{E}$ & NR & NIER & $\mathrm{T} / \mathrm{REF}$ \\
\hline \multirow[t]{2}{*}{$\mathrm{PB}^{1}$} & $\mathrm{R}$ & 0,38 & - & - & - & - & - & - & - & - & - & - & - & - \\
\hline & $\mathrm{P}$ & ns & - & - & - & - & - & - & - & - & - & - & - & - \\
\hline \multirow[t]{2}{*}{$\mathrm{TP}$} & $\mathrm{R}$ & $-0,08$ & $-0,56$ & - & - & - & - & - & - & - & - & - & - & - \\
\hline & $\mathrm{P}$ & $\mathrm{ns}$ & $*$ & - & - & - & - & - & - & - & - & - & - & - \\
\hline \multirow[t]{2}{*}{ TO } & $\mathrm{R}$ & 0,07 & 0,62 & $-0,83$ & - & - & - & - & - & - & - & - & - & - \\
\hline & $\mathrm{P}$ & ns & $*$ & $* *$ & - & - & - & - & - & - & - & - & - & - \\
\hline \multirow[t]{2}{*}{ TR } & $\mathrm{R}$ & $-0,30$ & 0,03 & $-0,80$ & 0,51 & - & - & - & - & - & - & - & - & - \\
\hline & $\mathrm{P}$ & ns & ns & $* *$ & ns & - & - & - & - & - & - & - & - & - \\
\hline \multirow[t]{2}{*}{ TB } & $\mathrm{R}$ & 0,008 & $-0,07$ & 0,07 & 0,16 & $-0,003$ & - & - & - & - & - & - & - & - \\
\hline & $\mathrm{P}$ & ns & $\mathrm{ns}$ & ns & ns & ns & - & - & - & - & - & - & - & - \\
\hline \multirow[t]{2}{*}{$\mathrm{NB} / \mathrm{E}$} & $\mathrm{R}$ & 0,62 & 0,82 & $-0,43$ & 0,62 & $-0,10$ & 0,20 & - & - & - & - & - & - & - \\
\hline & $\mathrm{P}$ & $* *$ & $* *$ & ns & $*$ & ns & ns & - & - & - & - & - & - & - \\
\hline \multirow[t]{2}{*}{ NEST/M } & $\mathrm{R}$ & $-0,52$ & $-0,70$ & 0,30 & $-0,27$ & 0,19 & 0,61 & $-0,61$ & - & - & - & - & - & - \\
\hline & $\mathrm{P}$ & ns & $* *$ & ns & ns & ns & $*$ & $*$ & - & - & - & - & - & - \\
\hline \multirow[t]{2}{*}{ TD } & $\mathrm{R}$ & $-0,43$ & $-0,77$ & 0,31 & $-0,39$ & 0,20 & $-0,005$ & $-0,80$ & 0,67 & - & - & - & - & - \\
\hline & $\mathrm{P}$ & ns & $* *$ & ns & ns & ns & ns & $* *$ & $* *$ & - & - & - & - & - \\
\hline \multirow[t]{2}{*}{$\mathrm{T} / \mathrm{E}$} & $\mathrm{R}$ & 0,52 & 0,58 & $-0,24$ & 0,15 & $-0,20$ & 0,72 & 0,47 & $-0,97$ & $-0,53$ & - & - & - & - \\
\hline & $\mathrm{P}$ & ns & $*$ & ns & $\mathrm{ns}$ & ns & $* *$ & ns & $* *$ & ns & - & & - & - \\
\hline \multirow[t]{2}{*}{ NR } & $\mathrm{R}$ & $-0,21$ & 0,14 & 0,24 & $-0,29$ & $-0,21$ & 0,29 & $-0,05$ & 0,14 & $-0,15$ & $-0,18$ & - & - & - \\
\hline & $\mathrm{P}$ & ns & ns & ns & ns & ns & ns & ns & ns & ns & ns & - & - & - \\
\hline \multirow[t]{2}{*}{ NIER } & $\mathrm{R}$ & 0,003 & 0,45 & $-0,13$ & 0,21 & 0,02 & 0,54 & 0,27 & 0,13 & $-0,32$ & $-0,28$ & 0,52 & - & - \\
\hline & $\mathrm{P}$ & ns & ns & ns & ns & ns & ns & ns & ns & ns & ns & ns & - & - \\
\hline \multirow[t]{2}{*}{ T/REF } & $\mathrm{R}$ & $-0,02$ & $-0,46$ & 0,24 & $-0,09$ & $-0,07$ & 0,05 & $-0,19$ & 0,32 & 0,28 & $-0,30$ & $-0,62$ & $-0,38$ & - \\
\hline & $\mathrm{P}$ & ns & ns & ns & ns & $\mathrm{ns}$ & ns & ns & ns & ns & ns & $*$ & ns & - \\
\hline \multirow[t]{2}{*}{ PLF } & $\mathrm{R}$ & 0,07 & 0,72 & $-0,70$ & 0,58 & 0,41 & $-0,05$ & 0,48 & $-0,44$ & $-0,60$ & 0,36 & 0,21 & 0,36 & $-0,71$ \\
\hline & $\mathrm{P}$ & ns & $* *$ & $* *$ & $*$ & ns & ns & $\mathrm{ns}$ & ns & $*$ & ns & ns & ns & $* *$ \\
\hline
\end{tabular}

${ }^{\mathrm{l}}$ Digestibilidade in vitro da matéria seca (DIVIMS), proteína bruta (PB), proporção de lâmina foliar (PLF), tempo de pastejo (TP), tempo de ruminação (TR) e tempo em ócio (TO), taxa de bocados (TB), número de bocados por estação (NB/E), número de estações por minuto (NEST/M), taxa de deslocamento (TD), tempo por estação (T/E) número de refeições $(\mathrm{NR})$, número de intervalos entre refeições (NIER) e tempo por refeição $(\mathrm{T} / \mathrm{REF})$ de bovinos em pastos de capim-piatã. $\mathrm{R}=$ coeficiente de correlação, $\mathrm{P}=* *$ e $*$ Significativo a 1 e $5 \%$ de probabilidade, respectivamente. 
Rev. Bras. Saúde Prod. Anim., Salvador, v.17, n.3, p. 385-400 jul./set., $2016 \quad \underline{\text { http://www.rbspa.ufba.br }}$ ISSN 15199940

A estrutura e o valor nutritivo do capimpiatã são afetados pelas alturas de entrada, que modificam o padrão de deslocamento, uso de estações alimentares e taxa de bocados dos animais. Assim, os resultados mostram que para se obter melhoria no comportamento ingestivo em pastos de capim-piatã os mesmos devem serem manejados entre $31,6 \pm 0,9$ a $41,7 \pm 0,8 \mathrm{~cm}$ de altura e com resíduo pós-pastejo de $23,4 \pm 1,5$ a $25,2 \pm 1,5 \mathrm{~cm}$.

\section{AGRADECIMENTOS}

Ao CNPq pelo apoio financeiro e a CAPES pela concessão da bolsa de estudos.

\section{REFERÊNCIAS}

CARLOTO, M.N.; EUCLIDES, V.P.B.; MONTAGNER, D.B.; LEMPP, B.; DIFANTE, G.S.; PAULA, C.C.L. Desempenho animal e características de pasto de capim-xaraés sob diferentes intensidades de pastejo, durante o período das águas. Pesquisa Agropecuária Brasileira, v.46, n.1, p.97-104, 2013.

CARVALHO, P.C.F.; TRINDADE, J.K.; MEZZALIRA, J.C.; POLI, C.H.E.C.; NABINGER, C.; GENRO, T.C.M.; GONDA, H.L. Do bocado ao pastoreio de precisão: compreendendo a interface planta animal para explorar a multifuncionalidade das pastagens. Revista Brasileira de Zootecnia, v.38, supl. especial, p.109-122, 2009.

CARVALHO, P.C.F.; GONDA, H.L.; WADE, M.H. Características estruturais do pasto e o consumo de forragem: o que pastar, quanto pastar e como mover para encontrar o pasto. In: MANEJO ESTRATÉGICO DA PASTAGEM, 4,
2008, Viçosa, MG. Anais... Viçosa, MG: Universidade Federal de Viçosa, 2008. p.101-130.

CASAGRANDE, D.R.; RUGGIERI, A.C.; JANUSCKIEWICZ, E.R.; GOMIDE, J.A.; REIS, R.A.; VALENTE, A.L.S.S. Características morfogênicas e estruturais do capimmarandu manejado sob pastejo intermitente com diferentes ofertas de forragem. Revista Brasileira de Zootecnia, v.39, n.10, p.2108-2115, 2010 .

DIFANTE, G.S.; EUCLIDES, V.B.P.; NASCIMENTO JUNIOR, D.; SILVA, S. C. da; TORRES JÚNIOR, R.A.A.; SARMENTO, D.O.L. Ingestive behaviour, herbage intake and grazing efficiency of beef cattle steers on tanzânia guineagrass subjected to rotational stocking managements.

Revista Brasileira de Zootecnia, v.38, n.6, p.1001-1008, 2009.

EUCLIDES, V.P.B.; MACEDO, M.C.M.; VALLE, C.B.; DIFANTE, G.S.; BARBOSA, R.A.; CACERE, E.R. Valor nutritivo da forragem e produção animal em pastagens de Brachiaria brizantha. Pesquisa Agropecuária Brasileira, v.44, n.1, p.98-106, 2009.

FACURI, L.M.A.M.; SILVA, R.R.; SILVA, F.F; CARVALHO, G. G.P.; SAMPAIO, C.B.; MENDES, F.B.L; LISBOA, M.M.; BARROSO, D.S.; CARVALHO, V.M.; PEREIRA, M.M.S. Ingestive behavior of heifers supplemented with glycerin in substitution of corn on Brachiaria brizantha Pasture. Asian-Australasian Journal of Animal Sciences, v.27, n.11, p.1584-1592, 2014. 
Rev. Bras. Saúde Prod. Anim., Salvador, v.17, n.3, p. 385-400 jul./set., $2016 \quad \underline{\text { http://www.rbspa.ufba.br }}$ ISSN 15199940

FLORES, R.S.; EUCLIDES, V.P.B.; ABRÃO, M.P.C.; GALBEIRO, S.; DIFANTE, G.S.; BARBOSA, R.A. Desempenho animal, produção de forragem e características estruturais dos capins marandu e xaraés submetidos a intensidades de pastejo. Revista Brasileira de Zootecnia, v.37, n.8, p.1355-1365, 2008.

FONSECA, L.; CARVALHO, P.C.F.; MEZZALIRA, J.C.; BREMM, C.; GALLI, J.R.; GREGORINI, P. Effect of sward surface height and level of herbage depletion on bite features of cattle grazing Sorghum bicolor swards. Journal of Animal Science, v.91, n.9, p.4357-4365, 2013.

GONÇALVES, E.N.; CARVALHO, P.C.F.; DEVINCENZI, T.; LOPES, M.L.T.; FREITAS, F.K.; JACQUES, A.V.A. Relações planta-animal em ambiente pastoril heterogêneo: padrões de deslocamento e uso de estações alimentares. Revista Brasileira de Zootecnia, v.38, n.11, p.2121-2126, 2009.

GREGORINI, P.; GUNTER, S.A.; BOWMAN, M.T.; CALDWELL, J.D.; MASINO, C.A.; COBLENTZ, W.K.; BECK, P.A. Effect of herbage depletion on short-term foraging dynamics and diet quality of steers grazing wheat pastures. Journal of Animal Science, v.89, n.11, p.3824-3830, 2011.

GRIFFITHS, W.M.; HODGSON, J.; ARNOLD, G.C. The influence of sward canopy structure on foreging descisions by grazing cattle. I. Regulation of bite depth. Grass and Forage Science, v.58, n.7, p. 125-137, 2003.

HOLDEN, L.A. Comparison of methods of in vitro matter digestibility for ten feeds. Journal Dairy Science, v.88, n.2, p.1791-1794, 1999.
KÖPPEN, W. Climatologia:

conunestudio de los climas de latierra. México: Fundo de Cultura Econômica, 1948. 479p.

MEZZALIRA, J.C.; BREMM, C.; TRINDADE, J.K.; NABINGER, C.; CARVALHO, P.C.F. The Ingestive behaviour of cattle in large-scale and its application to pasture management in heterogeneous pastoral environments. Journal of Agricultural Science and Technology, v.2, n.7, p.909-916, 2012.

MEZZALIRA, J.C.; CARVALHO, P.C.F.; AMARAL, M.F.; BREMM, C.; TRINDADE, J.K.; GONÇALVES, E.N.; GENRO, T.C.M.; SUÑÉ, R.W. Manejo do milheto em pastoreio rotativo para maximizar a taxa de ingestão por vacas leiteiras. Arquivos Brasileiros de Medicina Veterinária e Zootecnia, v.65, n.3, p.833-840, 2013.

MEZZALIRAA, J.C.; CARVALHO, P.C.F.; FONSECAA, L.; BREMMA, C.; CANGIANOB, C.; GONDAC, H.L.; LACA, E.A. Behavioral mechanisms of intake rate by heifers grazing swards of Contrasting structures. Applied Animal Behaviour Science, v.153, n.1, p.1-9, 2014.

OLIVEIRA NETO, R.A.; SILVA, J.H.Z.; ROCHA, M.G.; PÖTTER, L.; SICHONANY, M.J.O.; BISCAÍNO, L.L.; SANTOS, F.A.; BECK DIFANTE, M.V. Ingestive behavior, performance and forage intake by beef heifers on tropical pasture systems. Revista Brasileira de Zootecnia, v.42, n.8, p.549-558, 2013.

PALHANO, A.L., CARVALHO, P.C.F.; DITTRICH, J.R.; MORAES, A.; SILVA, S.C.; MONTEIRO, A.L.G. Padrões de deslocamento e procura por forragem de novilhas leiteiras em pastagem de capimmombaça. Revista Brasileira de Zootecnia, v.35, n.6, p.2253-2259, 2006. 
Rev. Bras. Saúde Prod. Anim., Salvador, v.17, n.3, p. 385-400 jul./set., $2016 \quad \underline{\text { http://www.rbspa.ufba.br }}$

SILVA, D.J.; QUEIROZ, A.C. Análises de alimentos: métodos químicos e biológicos. 3.ed. Viçosa, MG:

Universidade Federal de Viçosa, 2002. $235 p$.

TEIXEIRA, F.A.; BONOMO, P.; VIEIRA PIRES, A.J.V.; SILVA, F.F.; MARQUES, J.A.; SANTANA JÚNIOR, H.A. Padrões de deslocamento e permanência de bovinos em pastos de Brachiaria decumbens diferidos sob quatro estratégias de adubação. Revista Brasileira de Zootecnia, v.40, n.7, p.1489-1496, 2011.

TILLEY, J.M.A.; TERRY, R.A. A twostage technique for the in vitro digestion of forage crops. Journal Britani

Grasslands Society, v.18, n.2, p.104111, 1963.

WOLFINGER, R. Covariance structure selection in general mixed models.

Communications in Statistics Simulation, v.22, n.4, p.1079-1106, 1993.

Data de recebimento: $25 / 08 / 2015$

Data de aprovação: 19/07/2016 